\title{
Cost Evaluation of Endovascular Coiling and Surgical Clipping of Intracranial Aneurysm: A Systemic Review
}

\author{
Taiwo Akhigbe ${ }^{1 *}$, Ardalan Zolnourian ${ }^{1}$, Saadi Fahad ${ }^{2}$ \\ ${ }^{1}$ Department of Neurosurgery, University Hospital Southampton, Southampton, UK \\ 2 Altnalgelvin Area Hospital, Northern Ireland, UK
}

Corresponding Author: Taiwo Akhigbe, MBBS, MCh, MSc, PgD, Department of Neurosurgery, University Hospital Southampton, Tremona Rd, Southampton SO16 6YD, UK. E-mail: akhigbetaiwo@yahoo.com

Received October 22, 2016; Accepted November 8, 2016; Online Published December 2, 2016

\begin{abstract}
Introduction: Cost-effectiveness of medical intervention is becoming increasingly important in healthcare delivery. Treatment in neurosurgery is extremely expensive and there have been very few publications on neurosurgical health economics and comparative effectiveness analysis of neurosurgical procedures. Previous studies which had compared the costs of clipping and coiling were held out in European centers with data specifically from the ISAT study conducted in Europe. No significant differences were seen in the total cost of coiling when compared to clipping in a time period of one year.

Methods: A systematic literature search was conducted using electronic databases including MEDLINE and EMBASE. The inclusion criteria included the literature search limited to the last 10 years (2006-2016), studies on adult human patients, and papers published in English. All editorials, comments and correspondences were excluded from this study.

Results: Systematic literature search yielded 5784 studies. After removing duplicates and non-relevant studies, finally five studies had specifically analyzed cost economics of coiling and clipping of intracranial aneurysm. Overall, coiling was more expensive than clipping in three studies. The study which was conducted by Wolsteholme et al. showed no significant differences between the two procedures in terms of their costs.

Conclusion: Cost evaluations of intracranial aneurysm showed that surgical clipping results to reduced re-operation rate when compared to endovascular coiling, associated with increased complications, longer hospital stays and ultimately greater hospital costs.

Keywords: Cost-Effectiveness; Intracranial Aneurysm; Endovascular Coiling; Surgical Clipping

Citation: Akhigbe T, Zolnourian A, Fahad F. Cost evaluation of endovascular coiling and surgical clipping of intracranial aneurysm: a systemic review. Int J Med Rev. 2017;4(1):32-36. doi: 10.29252/ijmr-040106.
\end{abstract}

\section{Introduction}

The standard treatment of intracranial aneurysms has been neurosurgical clipping. However, with the introduction of detachable coil to treat aneurysm, endovascular coiling has currently emerged as a valid and reliable alternative to surgical clipping in the treatment of patients with Subarachnoid Aneurysmal Haemorrhage (SAH) and un-ruptured Intracranial Aneurysms (IAs.). ${ }^{1-3}$ The major advantage of endovascular coiling is the avoidance of craniotomy and early recovery following the procedure. The major disadvantage is in the event of coil impaction of the failed endovascular coiling. Actually, patients will have to undergo craniotomy. ${ }^{4}$ Endovascular coiling of intracranial aneurysm was firstly introduced in 1991 as an alternative to microsurgical clipping. The proportion of patients undergoing endovascular coiling increased from $34 \%$ to $54 \%$ in the UK after this publication. ${ }^{5}$

Cost-effectiveness of medical interventions is becoming increasingly important in healthcare delivery. Treatment in neurosurgery is becoming extremely expensive and there have been very few publications on neurosurgical health economics and comparative effectiveness analysis of neurosurgical procedures. ${ }^{6-9}$ Previous studies which had compared the cost of clipping and coiling were from European centers with data specifically from the ISAT study conducted in Europe. No significant differences were observed in the total cost of coiling when compared to clipping during a one year period. ${ }^{10,11}$

The aim of this review is to evaluate and analyses the cost-effectiveness of endovascular coiling (in comparison to neurosurgical clipping) in the treatment of intracranial aneurysm.

Copyright (C) 2016 The Author(s). This is an open-access article distributed under the terms of the Creative Commons Attribution License (http://creativecommons.org/licenses/by/4.0), which permits unrestricted use, distribution, and reproduction in any medium, provided the original work is properly cited. 


\section{Methods}

Systematic literature search was conducted using electronic databases including Medline, EMBASE and Google Scholar following the Preferred Reporting Items for Systematic Reviews and Meta-analyses (PRISMA). The search keywords were used individually and /or in combination to identify relevant papers. The used keywords included, Intracranial Aneurysm, Endovascular Coiling, Cost/ Cost-effectiveness. The inclusion criteria included the literature searches limited to the last 10 years (2006-2016), studies on adult human patients, and papers published in English. In addition, editorials, comments and correspondences were excluded. Finally, only five studies were used for this systematic review (Figure 1).

\section{Results}

Systematic literature search yielded 5784 studies. After removing duplicates and non-relevant studies, finally five studies that specifically analysed cost economics of coiling and clipping of intracranial aneurysm were used in this study (Figure 1). Overall, coiling was more expensive than clipping in three studies. ${ }^{12-14}$ The study conducted by Wolstenholme ${ }^{15}$ showed that clipping is much more expensive than coiling (Table 1). Treatment effectiveness was measured in QALY. Cost-effectiveness analysis includes additional consumed resources and associated health intervention measured in ICER (measure of the additional cost per health gain unit).

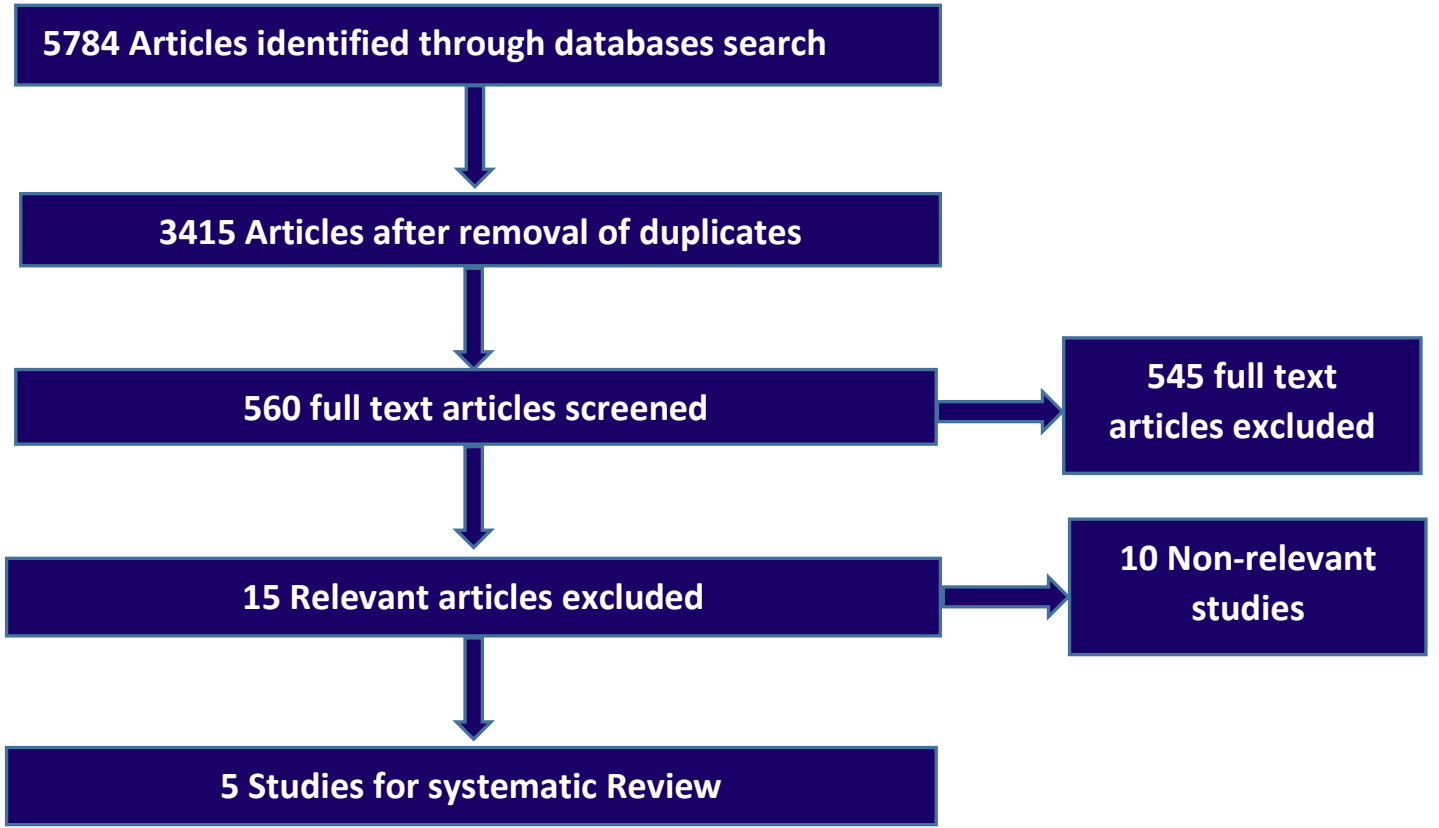

Figure 1. Search flow diagram

Table 1. Study Characteristics

\begin{tabular}{lccc}
\hline Study/ Year & Design & Clipping (cost) & Coiling (cost) \\
\hline Egger ${ }^{13} \mathbf{2 0 0 9}$ & Retrospective study & $\$ 41769$ & $\$ 45493$ \\
Wolstenholme $^{15} \mathbf{2 0 0 7}$ & Post randomisation analysis & $£ 20176$ & $£ 18436$ \\
\hline Tahir ${ }^{14} \mathbf{2 0 0 9}$ & Retrospective & $\$ 3127$ & $\$ 5080$ \\
Lindsay $^{16} \mathbf{2 0 1 0}$ & Retrospective & $\$ 169$ 942 (Ruptured) $\$ 80893$ & $\$ 130,462$ (Ruptured) \\
& & (Un-ruptured) & $\$ 68644$ (Unuptured) \\
Lad $^{12} \mathbf{2 0 1 3}$ & Retrospective & $\$ 79577$ & $\$ 82986$ \\
\hline
\end{tabular}

\section{Discussion}

A cost-effectiveness analysis was carried out by Maud et al. ${ }^{13}$ between the endovascular and neurosurgical treatment for ruptured intracranial aneurysms in the United States. The clinical data and values for quality of life were extracted from the ISAT trial. ${ }^{17}$ Patients' eligibility were determined by the presence of a ruptured intracranial aneurysm judged by a neurosurgeon and interventional neuroradiologist to be suitable for either coiling or clipping based on its angiographic anatomy as agreed by both parties. Costs were derived from data on long-term care of stroke patients, relevant literatures and the largest hospital databases. Cost analysis include the total cost of hospitalisation, disability cerebral angiography, frequency of retreatment and rebreeding for aneurysmal subarachnoid haemorrhage in US in 2005-2006 using the Prospective Comparative DatabaseTreatment. Cost-effectiveness analysis includes additional resources consumed and associated with health intervention measured in ICER (measure of the additional cost per health gain unit). The average cost was estimated by multiplying each cost by the rate of clinical outcomes from the findings of ISAT. 
This research was a retrospective study based on the foremost database in the United States from 2005-2006 using the ISAT trial to extract data and for comparisons. ISAT is the best evidence for the treatment of intracranial aneurysm. About 1000 patients were randomized in each group with the majority in good clinical conditions (WFNS Grades I-III). Initial hospitalization of endovascular coiling of low severity case for subarachnoid haemorrhage costs $\$ 35,143$ while moderate to severe disability case costs $\$ 21,645$ with a median cost of $\$ 3370$. The median cost of cerebral angiogram was $\$ 2800$ and the retreatment and rebreeding cost were $\$ 21,920$ and $\$ 61,622$ respectively. The average cost per patient for a 1 year period with ruptured intracranial aneurysm treated by endovascular coiling was $\$ 45,493$. Meanwhile, in the neurosurgical group, the initial hospitalisation of low severity SAH case was $\$ 35,005$. Also, the cost of retreatment and rebreeding were $\$ 25,150$ and $\$ 58,426$ respectively. The average cost per patient for a 1 year period with an intracranial aneurysm treated by clipping was $\$ 41,769$. The overall QALY calculated for coiling and clipping were 0.69 and was 0.64 respectively. The mean ICER for coiling and clipping was \$72,872 per QALY. Higher costs were recorded in the endovascular group in a one year period due to the retreatment of the originally treated intracranial aneurysm.

Cost analysis for endovascular coiling and surgical clipping may not reflect the exact cost for hospitalisation and services because these were collected retrospectively and this may be influenced by ascertainment and documentation biases. In addition, the endovascular treatment is evolving at a rapid rate with significant and remarkable changes and adjustment. Hence, this will go a long way to influence the cost effectiveness unlike surgical clipping technique with little or no changes. This study reveals that in the United States, endovascular coiling results with higher costs in a one year period. It also associates with better outcomes compared to surgical clipping among patients with ruptured intracranial aneurysm suitable for either endovascular or neurosurgical treatment pathways.

Wolstenholme et al. ${ }^{15}$ carried out a cost analysis on the treatment pathways, used resources and the cost of endovascular coiling and surgical clipping after aneurysmal subarachnoid haemorrhage. This study was a post-randomisation analysis of ISAT. A total number of 2143 patients were recruited to the ISAT trial by 42 neurological centres. This is while this study focused on the UK patients only comprising 1644 patients with a randomised endovascular group of 809 patients and a neurosurgical group of 835 patients. These patients were followed up for two years.

It was obviously seen that during the first episode of care, the staff costs associated with interventions were remarkably lower in the endovascular group $(£ 1450$ per patient) as compared to the neurosurgical group ( $£ 2108)$, a difference of $£ 658$. As the consumable costs were higher in the endovascular group ( $\mathfrak{E}$ 2627) than the neurosurgical group (£901), this significant difference was due to the cost of coils. The costs of post-operative length of stay in the first episode of care were much lower in the endovascular group ( $£ 11$ 547) compared to the neurosurgical group (£15 311). These results reveal that neurosurgical groups spent more days in the inpatient wards, ITU and rehabilitation clinics compared to the endovascular. The mean and standard deviation of the overall total cost per patients after 12-months follow up was estimated to be $£ 18436$ and $£ 15849$ respectively for the endovascular group. This is while, the mean value of $£ 20176$ with a standard deviation of £21 984 for the neurosurgical group with insignificant difference of $£ 1740$ was in favour of the endovascular patients. Furthermore, subsequent care costs from 12 to 24 months follow up showed a mean cost of $£ 613$ in the endovascular group as against $\mathfrak{£} 131$ in the neurosurgical group. This actually shows a remarkable difference of $£ 482$ in favour of the neurosurgical group.

There is no significant difference in the treatment costs for aneurysmal subarachnoid haemorrhage, though in favour of endovascular group in the first 12 months, endovascular patients had higher cost of treatment during the first intervention that was latter minimized by length of stay. In addition, in the 12 to 24 months follow up period, costs were greater in the endovascular group due to subsequent procedures including angiograms, complications and adverse effects. There is no information regarding the costs for long-term nursing and informal care hence this study is not a reflection of accurate and exact cost effectiveness. However, it provides a significant glimpse into the cost economics of aneurysmal subarachnoid treatment pathway.

Tahir et al. ${ }^{14}$ performed a study on the cost-effectiveness of clipping compared to coiling of intracranial aneurysm after subarachnoid haemorrhage in a developing country. This research was a prospective study with a population of 55 patients with aneurysmal subarachnoid haemorrhage and was actively treated and followed up for ruptured aneurysm at Aga Khan University Hospital (Pakistan) between January 2004 and June 2007.

All costs were in Pakistani Rupees (Rs) which got converted to US dollars, $60 \mathrm{Rs}=\$ 1$ ). Costs included treatment costs (clipping or coiling), inpatient stay (initial stay, regular bed, ICU bed occupancy), and radiologic follow up (angiograms). Total costs were manually calculated. Clinical outcomes were measured at the time of discharged and six months follow up visit using the modified Rankin scale. Statistical analysis were performed using SPSS 13.0 (SPSS, Chicago, and III). The average total cost for coiling and clipping was $\$ 5080$ (Rs. 304800 ) and $\$ 3127$ (Rs. 187620 ) respectively. The cost of coiling was found to be $62 \%$ more expensive compared to the cost of clipping without providing additional benefit of decrease morbidity. There was a decrease in the cost of hospital stay in the endovascular group due to the short length of stay as compared to the surgical group. 
As this study was conducted in a developing country, only $7 \%$ of them were medically insured. This issue put huge financial pressure on both patients and their relatives. In terms of the clinical outcome, this study showed no significant difference between coiling and clipping.

Hol et al. ${ }^{18}$ conducted a study on the length of stay and total hospital charges of clipping versus coiling for ruptured and un-ruptured Adult Cerebral Aneurysms in the Nationwide Inpatient Sample Database that is the largest all-payer inpatient care database in America, containing hospitalizations for clipping or coiling of ruptured and un-ruptured cerebral aneurysms from 2002 to 2006. A comparison was done between the length of hospital stay and total hospital charges for clipping and coiling. Accordingly, 9635 hospitalizations for ruptured aneurysm treatments (6019 clipping, 3616 coiling) and 9399 hospitalizations for un-ruptured aneurysm treatments (4700 clipping, 4699 coiling) were measured. After considering all the relevant hospital and patient characteristics, on average, clipping resulted in \$15 325 more in total charge for ruptured patients and resulted in $\$ 11263$ more in total charge for un-ruptured patients. While comparing clipping to coiling after the adjustment for all-related factors, clipping had an average of 1.2 times more days in hospitalization for ruptured patients and was associated with an average of 1.8-times more days in hospitalization for un-ruptured patients. After considering all relevant hospital and patient characteristics, on average, clipping resulted in $\$ 15325$ more in total charge for ruptured patients and resulted in \$11 263 more in total charge for un-ruptured patients. The results of the nationwide analysis differed from this single-institution study. Clipping was faced with significantly longer lengths of stay and also higher total hospital charges for both ruptured and un-ruptured aneurysm patients in America over these four over period in study compared to coiling.

A retrospective study was performed by Lad et al. ${ }^{12}$ on the long-term economic impact of coiling versus clipping for un-ruptured intracranial aneurysms. Market-Scan database (which is a collection of six different databases) was utilised to identify and examine patients who had either endovascular coiling or surgical clipping procedures for un-ruptured intracranial aneurysm for a 9-year period (from 2000 to 2009) comparing health care resource use, angiogram, complications and reoperation rates. Market-Scan is all comprehensive and allinclusive.

A total of 4504 patients were involved in this study. Among them, 1878 underwent clipping while 2626 patients had coiling. The used healthcare resources include length of stay during initial procedure hospitalization, accumulated days of subsequent hospitalizations and postoperative outpatient services at

\section{References}

1. Broadbent LP, Moran CJ, Cross DT, 3rd, Derdeyn CP. Management of neuroform stent dislodgement and one, two and five years, medications prescription and ED services with primary diagnosis of un-ruptured intracranial aneurysm. The total costs were calculated as a sum of in-hospital costs, outpatients cost and medication costs.

The total cost for patients who underwent clipping was $\$ 79577$ compared to $\$ 82986$ for the endovascular group $(\mathrm{P}=0.69)$, aneurysm-related ( $\$ 60945$ vs. $\$ 63835, \mathrm{P}=$ 0.53 ) and haemorrhage related ( $\$ 1257$ vs $\$ 2632 . \mathrm{P}=$ 0.27 ). During a 2 year period, patients who underwent clipping and coiling had similar overall outcomes ( $\$ 74$ 294 vs $\$ 77791, P=0.85$ ), aneurysm related ( $\$ 58039$ vs. $\$ 61999, \mathrm{P}=0.71)$ and haemorrhage related ( $\$ 1731$ vs $\$ 1808, \mathrm{P}=0.63$ ) health costs. There was no significant differences at 5-year overall total healthcare cost of $\$ 75$ 784 for surgical clipping compared to $\$ 73673$ for endovascular coiling $(P=0.51)$. Patients who underwent surgical clipping accumulated more haemorrhage related costs at 5 years ( $\$ 3352$ vs $\$ 731, \mathrm{P}=0.63)$. Though clipping is associated with increased complications but results in less reoperation rates hence, the cost of clipping and coiling are comparable at 5-yrears. This is while those in the coiling group accumulate more costs due to longer follow up as a result of high reoperation rates and angiogram rates. The study conducted by Maud et al. was the only research which recorded QUALY and ICER values ${ }^{13}$.

\section{Conclusions}

Cost economics of intracranial aneurysm showed that surgical clipping reduced the re-operation rate when compared to endovascular coiling and also associated with increased complication, longer hospital stay and ultimately greater hospital cost. However, clipping is costlier than coiling for the initial procedure but in terms of the overall cost for one, two and five years post procedure period did not reveal any statistically significant differences. In addition, Wolstenholme ${ }^{15}$ found no significant difference in regards to the cost between coiling and clipping 2-years post aneurysm treatment. However, an Australian studyl showed that clipping was associated with higher total costs.

\section{Ethical Approval}

None.

\section{Acknowledgment}

None.

\section{Authors' Contributions}

All authors contributed equally to this study.

\section{Conflict of Interest Disclosures}

No conflicts of interest.

\section{Funding/Support}

None.

misplacement. AJNR Am J Neuroradiol. 2003;24(9):181922. pmid: 14561609 . 
2. Egger $M$, Zellweger-Zahner $T$, Schneider $M$, Junker $C$, Lengeler C, Antes G. Language bias in randomised controlled trials published in English and German. Lancet. 1997;350(9074):326-9. doi: 10.1016/S0140-6736(97)0241 9-7. pmid: 9251637.

3. Hwang JS, Hyun MK, Lee HJ, Choi JE, Kim JH, Lee NR, et al. Endovascular coiling versus neurosurgical clipping in patients with unruptured intracranial aneurysm: a systematic review. BMC Neurol. 2012;12:99. doi: 10.1186/1471-237712-99. pmid: 22998483.

4. Bairstow P, Dodgson A, Linto J, Khangure M. Comparison of cost and outcome of endovascular and neurosurgical procedures in the treatment of ruptured intracranial aneurysms. Australas Radiol. 2002;46(3):249-51. doi: 10.1046/j.1440-1673.2002.01053.x. pmid: 12196230.

5. Lindsay KW. The impact of the International Subarachnoid Aneurysm Treatment Trial (ISAT) on neurosurgical practice. Acta Neurochir (Wien). 2003;145(2):97-9. doi: 10.1007 /s00701-002-1066-7. pmid: 12601456.

6. Molyneux A, Kerr R, Stratton I, Sandercock P, Clarke M, Shrimpton J, et al. International Subarachnoid Aneurysm Trial (ISAT) of neurosurgical clipping versus endovascular coiling in 2143 patients with ruptured intracranial aneurysms: a randomised trial. Lancet. 2002;360(9342):1267-74. doi: 10.1016/S0140-6736(02)11 314-6. pmid: 12414200.

7. Naggara ON, White PM, Guilbert F, Roy D, Weill A, Raymond J. Endovascular treatment of intracranial unruptured aneurysms: systematic review and meta-analysis of the literature on safety and efficacy. Radiology. 2010;256(3):887-97. doi: 10.1148/radiol.10091982. pmid: 20634431.

8. Raymond J, Roy D, Bojanowski M, Moumdjian R, L'Esperance G. Endovascular treatment of acutely ruptured and unruptured aneurysms of the basilar bifurcation. J Neurosurg. 1997;86(2):211-9. doi: 10.3171/jns.1997.86.2 .0211. pmid: 9010422.

9. Raymond J, Guilbert F, Weill A, Georganos SA, Juravsky L, Lambert $A$, et al. Long-term angiographic recurrences after selective endovascular treatment of aneurysms with detachable coils. Stroke. 2003;34(6):1398-403. doi: 10.1161/01.STR.0000073841.88563.E9. pmid: 12775880.

10. van der Schaaf I, Algra A, Wermer M, Molyneux A, Clarke $M$, van Gijn J, et al. Endovascular coiling versus neurosurgical clipping for patients with aneurysmal subarachnoid haemorrhage. Cochrane Database Syst Rev.
2005(4):CD003085. doi: 10.1002/14651858.CD003085.pu b2. pmid: 16235314 .

11. Vanninen R, Koivisto T, Saari T, Hernesniemi J, Vapalahti M. Ruptured intracranial aneurysms: acute endovascular treatment with electrolytically detachable coils--a prospective randomized study. Radiology. 1999;211(2):32536. doi: 10.1148/radiology.211.2.r99ap06325. pmid: 10228510.

12. Lad SP, Babu R, Rhee MS, Franklin RL, Ugiliweneza B, Hodes J, et al. Long-term economic impact of coiling vs clipping for unruptured intracranial aneurysms. Neurosurgery. 2013;72(6):1000-11; discussion 11-3. doi: 10.1227/01.neu.0000429284.91142.56. pmid: 23612602.

13. Maud A, Lakshminarayan K, Suri MF, Vazquez G, Lanzino G, Qureshi Al. Cost-effectiveness analysis of endovascular versus neurosurgical treatment for ruptured intracranial aneurysms in the United States. J Neurosurg. 2009;110(5):880-6. doi: 10.3171/2008.8.JNS0858. pmid: 19199452.

14. Zubair Tahir M, Enam SA, Pervez Ali R, Bhatti A, ul Haq T. Cost-effectiveness of clipping vs coiling of intracranial aneurysms after subarachnoid hemorrhage in a developing country--a prospective study. Surg Neurol. 2009;72(4):35560; discussion 60-1. doi: 10.1016/j.surneu.2008.11.003. pmid: 19616277.

15. Wolstenholme J, Rivero-Arias O, Gray A, Molyneux AJ, Kerr RS, Yarnold JA, et al. Treatment pathways, resource use, and costs of endovascular coiling versus surgical clipping after aSAH. Stroke. 2008;39(1):111-9. doi: 10.1161/STROKEAHA .107.482570. pmid: 18048858.

16. Linn FH, Rinkel GJ, Algra A, van Gijn J. Incidence of subarachnoid hemorrhage: role of region, year, and rate of computed tomography: a meta-analysis. Stroke. 1996;27(4):625-9. doi: 10.1161/01.STR.27.4.625. pmid: 8614919 .

17. Campi A, Ramzi N, Molyneux AJ, Summers PE, Kerr RS, Sneade M, et al. Retreatment of ruptured cerebral aneurysms in patients randomized by coiling or clipping in the International Subarachnoid Aneurysm Trial (ISAT). Stroke. 2007;38(5):1538-44. doi: 10.1161/STROKEAHA.106.46698 7. pmid: 17395870.

18. Hoh BL, Chi YY, Lawson MF, Mocco J, Barker FG, 2nd. Length of stay and total hospital charges of clipping versus coiling for ruptured and unruptured adult cerebral aneurysms in the Nationwide Inpatient Sample database 2002 to 2006. Stroke. 2010;41(2):337-42. doi: 10.1161/STROKEAHA.109.569269. pmid: 20044522. 